\title{
Escoamento uniforme em canais circulares lisos. Parte I: Adaptação e validação do método de Kazemipour ${ }^{1}$
}

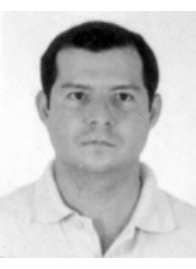

Maurício C. Goldfarb ${ }^{2}$, Tarciso C. da Silva ${ }^{3} \&$ Carlos A. V. de Azevedo ${ }^{4}$

\footnotetext{
1 Parte da Dissertação de Mestrado do primeiro autor apresentada na UFCG

2 DTCC/CT/UFPB. CEP 58059-900, João Pessoa, PB. Fone (83) 216-7037. E-mail: goldfarb@lrh.ct.ufpb.br (Foto)

${ }^{3}$ DTCC/CT/UFPB. Fone (83) 216-7684. E-mail: tarciso@lrh.ct.ufpb.br

${ }^{4}$ DEAg/CCT/UFCG. Av. Aprígio Veloso, 882, Bodocongó, CEP 58109-970, Campina Grande, PB. Fone: (83) 310-1056. E-mail: cazevedo@deag.ufcg.edu.br,
}

\author{
Protocolo $45-6 / 3 / 2003$ - Aprovado em 30/4/2004
}

\begin{abstract}
Resumo: A partir da equação de von Karman Prandtl para tubos pressurizados, Kazemipour \& Apelt (1980) desenvolveram uma metodologia para cálculo do escoamento em canais circulares lisos, denominada método de Kazemipour o qual, apesar de apresentar resultados de bastante eficiência necessita, no entanto, de recursos gráficos na sua aplicação, o que impossibilita a solução através de métodos computacionais e, também, a comparação deste com outras metodologias existentes. Neste trabalho, mostram-se os resultados da investigação analítica que resulta na validação do método de Kazemipour, como também o ajuste, de acordo com o procedimento proposto por Silva \& Figueiredo (1993), de maneira a tornar o procedimento completamente equacionável sem a necessidade de recursos gráficos. O resultado encontrado é satisfatório e sua aplicação é apresentada num exemplo de aplicação prática.
\end{abstract}

Palavras-chave: irrigação, vazão, hidráulica

\section{Uniform flow in smooth circular channels. Part I: Adaptation and validation of the Kazemipour method}

\begin{abstract}
Considering the von Karman Prandtl equation for pressurized tubes, Kazemipour \& Apelt (1980) developed a methodology for flow calculation in smooth circular channels, denominated as method of Kazemipour. Inspite of good results, the Kazemipour method needs graphic tools in its application, which makes its solution through computational methods and comparison to other existing methodologies difficult. In this research, the results of the analytic investigation that provides the validation of the Kazemipour method are shown, as well as the adjustments according to procedure proposed by Silva \& Figueiredo (1993), performed in such a way to make the procedure independent of graphic tools. The result obtained is satisfactory and its use is presented in an example of practical application.
\end{abstract}

Key words: Irrigation, discharge, hydraulics

\section{INTRODUÇÃO}

Os canais circulares são largamente utilizados tanto no meio rural, na condução de água para diversas aplicações e drenagem de terras agrícolas, como nas cidades, para drenagem pluvial e de esgotos domésticos e industriais. Um grande número de modelos hidráulicos que representam o escoamento turbulento uniforme em canais pode ser aplicado no dimensionamento dessas estruturas. Devido aos elevados custos financeiros que geralmente envolvem obras de canais hidráulicos, a adoção de um procedimento de cálculo eficaz pode proporcionar considerável economia no valor global empregado.

Trabalhando com canais circulares e retangulares, Kazemipour \& Apelt (1980) revisaram e analisaram os principais métodos de cálculo da resistência hidráulica ao escoamento uniforme. Constatando serem os métodos analisados incompletos ou falhos, empreenderam então uma investigação teórica apoiada nos dados experimentais de outros autores e nos resultados obtidos experimentalmente por eles mesmos.

Assim sendo, Kazemipour \& Apelt (1980) desenvolveram uma metodologia de bastante eficiência para o cálculo do 
escoamento em canais de seções transversais retangulares e circulares, denominada "método de Kazemipour". O "método de Kazemipour", que permite o cálculo do fator de atrito para canais em escoamento turbulento uniforme, tem sido considerado como uma eficiente metodologia por diversos autores, a exemplo de Nalluri \& Adepoju (1985), Oliveira (1994) entre outros. Diferente das metodologias tradicionais que representam a geometria da seção transversal através da área e do perímetro molhado, o método de Kazemipour, utiliza na representação da forma da seção, uma combinação de dois parâmetros distintos, ditos parâmetros de forma. O primeiro parâmetro de forma utilizado pode ser calculado através de uma relação analítica de grandezas da geometria do escoamento e o segundo parâmetro do método de Kazemipour, obtido dos valores experimentais do escoamento, é fornecido apenas de forma gráfica. O comportamento da curva que descreve o segundo parâmetro impede sua representação por equações corriqueiramente utilizadas na engenharia.

Através do ajuste numérico do método de Kazemipour, objetivou-se que o mesmo, quando utilizado no cálculo do escoamento em canais circulares lisos, seja completamente equacionável através de uma rotina computacional, o que possibilita não só a sua comparação com outras metodologias como, também, reduz os inevitáveis erros decorrentes das leituras de gráficos.

\section{MATERIAL E MÉTODOS}

$\mathrm{O}$ ajuste numérico, para representar matematicamente o segundo parâmetro do método de Kazemipour, é feito neste trabalho utilizando-se variáveis polinomiais transformadas, de acordo com a metodologia proposta por Silva \& Figueiredo (1993).

\section{O método de Kazemipour e sua validação analítica}

O método de Kazemipour representa os efeitos da forma da seção transversal do escoamento por meio de dois parâmetros adimensionais considerando-se, de maneira satisfatória, a desuniformidade da tensão de cisalhamento no perímetro molhado de canais circulares, apresentada por Repogle \& Chow (1966).

O primeiro parâmetro adimensional utilizado no método de Kazemipour para o cálculo do escoamento turbulento uniforme em canais circulares pode ser calculado pela Eq. 1 .

$$
\psi_{1}=(\mathrm{P} / \mathrm{D})^{1 / 2}
$$

donde, P é o perímetro molhado da seção transversal do escoamento e D o diâmetro interno do canal.

O segundo parâmetro do método de Kazemipour, $\psi_{2}$, é uma função do diâmetro interno do canal, $\mathrm{D}$, e da profundidade efetiva do escoamento, $y_{D}$, que representam a forma da geometria característica da seção transversal. O segundo parâmetro, $\psi_{2}$, foi escolhido de modo que a combinação desses dois parâmetros ajustasse os valores do fator de atrito para canais abertos, tornando-os iguais aos valores dos fatores de atrito para tubulações pressurizadas, para um mesmo número de Reynolds.
Os valores de $\psi_{2}$ assim obtidos foram plotados versus valor da relação $\mathrm{D} / \mathrm{y}_{\mathrm{D}}$, desta maneira, a curva que representa o melhor ajuste dos pontos foi traçada por Kazemipour \& Apelt (1980), conforme a Figura 1, a partir da qual o segundo parâmetro pode ser obtido entrando-se com o parâmetro geométrico $\mathrm{D} / \mathrm{y}_{\mathrm{D}}$, na curva apresentada.

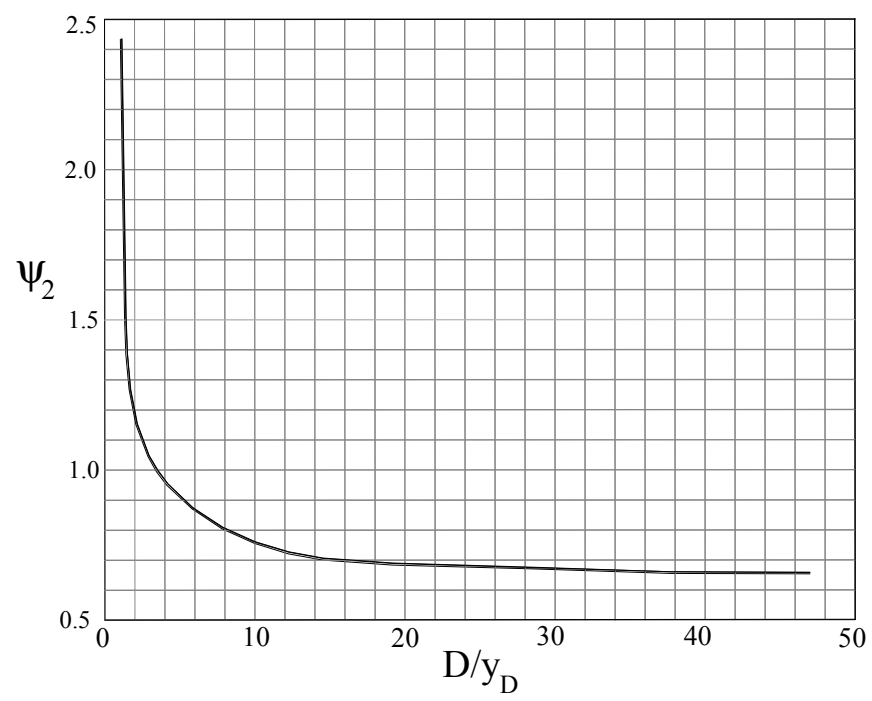

Figura 1. Curva do segundo parâmetro de forma do método de Kazemipour (Kazemipour \& Apelt, 1980)

Uma vez obtidos os dois parâmetros de forma, a razão entre essas grandezas fornece o parâmetro, $\psi$, que representa a geometria da seção transversal do escoamento. A Eq. 2 fornece a razão de cálculo deste parâmetro.

$$
\psi=\psi_{1} / \psi_{2}
$$

De posse do parâmetro de forma $\psi$, o cálculo do escoamento uniforme em canais de seção circular pode ser feito corrigindose o fator de atrito de modo a se poder utilizar as equações para tubos pressurizados, conforme metodologia apresentada por Kazemipour \& Apelt (1980).

Utilizando-se a metodologia proposta por Silva (1992) inicialmente para o cálculo do escoamento em canais retangulares, Goldfarb (2002) desenvolveu um modelo matemático baseado nas equações da quantidade de movimento, da continuidade, e na distribuição experimental da tensão de cisalhamento no perímetro molhado, obtida por Repogle \& Chow (1966). Desta forma, Goldfarb (2002) obteve curvas teóricoanalíticas no sentido de validar o método de Kazemipour. A Figura 2 apresenta as curvas do segundo parâmetro de forma, $\Psi_{2}$, que vem demonstrar a validade do método de Kazemipour, visto que as duas curvas diferem apenas por uma constante.

\section{Ajuste numérico do método de Kazemipour}

Para se adaptar o método de Kazemipour à formulação puramente algébrica, necessita-se da equação do segundo parâmetro de forma $\Psi_{2}$, não fornecida por Kazemipour \& Apelt (1980), apesar de ser o melhor ajuste aos pontos plotados na curva apresentada na Figura 1, cuja abscissa representa o diâmetro interno do canal, D, dividido pela profundidade efetiva 


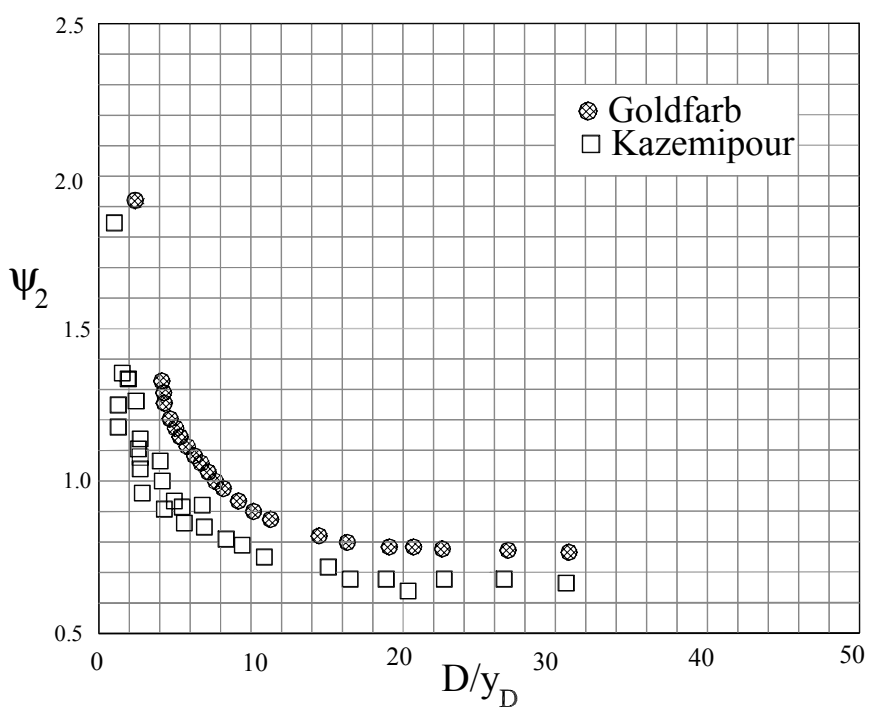

Figura 2. Segundo parâmetro de forma, $\psi_{2}$ (Goldfarb, 2002)

do escoamento, $\mathrm{y}_{\mathrm{D}}$, e a ordenada o segundo parâmetro de forma, $\psi_{2}$.

A obtenção da equação de $\psi$, resulta da utilização de variáveis polinomiais transformadas, de acordo com a metodologia proposta por Silva \& Figueiredo (1993). Neste procedimento, calculam-se, inicialmente, as variáveis dependente e independente, segundo as Eqs. (3) e (4).

$$
\begin{gathered}
\mathrm{X}=\mathrm{C}_{1}+\ln \left(\mathrm{D} / \mathrm{y}_{\mathrm{D}}\right) \\
\mathrm{Y}=\mathrm{C}_{2}+\ln \psi_{2}
\end{gathered}
$$

sendo $\mathrm{X}$ e $\mathrm{Y}$ variáveis auxiliares, respectivamente independente e dependente. A introdução das constantes $\mathrm{C}_{1}$ e $\mathrm{C}_{2}$ tem por finalidade evitar valores negativos de $\mathrm{X}$ e $\mathrm{Y}$. Neste caso, $\mathrm{C}_{1}=\mathrm{C}_{2}=1$.

De acordo com a metodologia de ajuste, as variáveis dependente e independente obtidas são correlacionadas através de uma função polinomial de grau n. De posse do polinômio, o segundo parâmetro do método de Kazemipour, $\psi_{2}$, pode ser então obtido através da Eq. (5), proposta por Silva \& Figueiredo (1993).

$$
\psi_{2}=\exp \left\{\left[\sum_{i=0}^{n} \quad \mathrm{a}(\mathrm{i})\left(\mathrm{C}_{1}+\ln \left(\mathrm{D} / \mathrm{y}_{\mathrm{D}}\right)\right)^{\mathrm{i}}\right]-\mathrm{C}_{2}\right\}
$$

sendo a(i) os coeficientes e n o grau do polinômio ajustado que relaciona as variáveis $\mathrm{X}$ e $\mathrm{Y}$.

\section{Procedimento para aplicação do método de Kazemipour}

De posse do diâmetro interno do canal, da altura hidráulica do escoamento, da declividade do fundo do canal e da temperatura da água, a vazão escoada em um canal circular liso pode ser obtida através do método de Kazemipour, de acordo com a seqüência de cálculo proposta por Silva (1992), inicialmente para cálculo do escoamento em canais retangulares. A seqüência de cálculo que torna o método de Kazemipour solucionável a partir de equações explícitas, possui a rotina de execução seguinte: i) Cálculo da área da seção transversal.

ii) Cálculo de $\mathrm{y}_{\mathrm{D}}\left(\mathrm{y}_{\mathrm{D}}=\mathrm{A} / \mathrm{D}\right)$.

iii) Cálculo de $\psi_{2}$ (Eq. 5) e obtenção de $\psi$ (Eqs. 1 e 2).

iv) Através da equação seguinte, apresentada por Silva (1992), calcula-se a parcela $\operatorname{Re}(f)^{1 / 2}$.

$$
\operatorname{Re}(f)^{1 / 2}=\left[128 \mathrm{R}^{3} \mathrm{gS} / \mathrm{n}^{2} \psi\right]^{1 / 2}
$$

donde Re é o número de Reynolds, $f$ o coeficiente de atrito para tubulações pressurizadas, $\mathrm{R}$ o raio hidráulico, $\mathrm{g}$ a aceleração da gravidade, $\mathrm{S}$ a declividade do fundo do canal, $v$ a viscosidade cinemática da água e $\psi$ o parâmetro de forma do método de Kazemipour.

v) Obtém-se o coeficiente de atrito para tubulações pressurizadas f da equação de von Karmam Prandtl para tubos pressurizados, de acordo com a Eq. (7).

$$
\frac{1}{\sqrt{f}}=2 \log \operatorname{Re} \sqrt{f}-0,8
$$

vi) Calcula-se o fator de atrito para canais $\mathrm{fc}=\mathrm{f} \psi$

vii) Calcula-se a velocidade da equação universal de perdas de carga

$$
V=\sqrt{\frac{8 g R S}{f c}}
$$

em que $V$ é a velocidade média no canal, $\mathrm{R}$ o raio hidráulico e $\mathrm{S}$ a declividade do fundo do canal.

viii) Finalmente, calcula-se a vazão $Q$ através do produto da área da seção transversal $\mathrm{A}$, pela velocidade média $V$.

\section{RESULTADOS E DISCUSSÃO}

Os dados de Kazemipour \& Apelt (1980) retirados da curva apresentada por esses autores (Figura 1), foram então lançados nas Eq. (3) e (4), conforme a metodologia de ajuste proposta por Silva \& Figueiredo (1993). Os valores assim obtidos fornecem os coeficientes do polinômio de ajuste, cujo coeficiente de correlação depende do grau do polinômio escolhido. A Tabela 1 apresenta os valores dos coeficientes para polinômios de grau $\mathrm{n}$ variando de 1 a 5 .

Os valores do coeficiente de correlação relativamente elevados, 0,8936, para um polinômio de grau 1 até 0,9994, para um polinômio de grau 5, observados na Tabela 1 , demonstram a potência da metodologia de ajuste adotada. Para a aplicação proposta, um coeficiente de correlação superior a 0,99 é considerado satisfatório; portanto, o polinômio de grau 2 já atente às necessidades de precisão para a equação do segundo parâmetro do método de Kazemipour, $\psi_{2}$, procurada. Neste caso, um polinômio de ordem superior a 2 poderia ter sido escolhido, mas o resultado encontrado não teria a simplicidade da equação obtida para o segundo parâmetro. 
Tabela 1. Valores dos coeficientes de polinômios de ajuste

\begin{tabular}{cccccc}
\hline $\mathrm{a}(\mathrm{i})$ & $\mathrm{n}=1$ & $\mathrm{n}=2$ & $\mathrm{n}=3$ & $\mathrm{n}=4$ & $\mathrm{n}=5$ \\
\hline $\mathrm{a}(0)$ & 1,605 & 2,312 & 2,853 & 3,827 & 5,596 \\
$\mathrm{a}(1)$ & $-0,243$ & $-0,764$ & $-1,413$ & $-2,986$ & $-6,619$ \\
$\mathrm{a}(2)$ & - & 0,084 & 0,318 & 1,198 & 4,012 \\
$\mathrm{a}(3)$ & - & - & $-0,026$ & $-0,231$ & $-1,264$ \\
$\mathrm{a}(4)$ & - & - & - & 0,017 & 0,198 \\
$\mathrm{a}(5)$ & - & - & - & - & $-0,012$ \\
$\mathrm{r}^{2}$ & 0,8936 & 0,9901 & 0,9963 & 0,9986 & 0,9994 \\
\hline
\end{tabular}

O polinômio escolhido com valor do coeficiente de correlação $\mathrm{r}^{2}=0,9901$, é apresentado na Eq. (9), relacionando-se as variáveis $\mathrm{X}$ e $\mathrm{Y}$, respectivamente dependente e independente.

$$
\mathrm{Y}=0,084 \mathrm{X}^{2}-0,764 \mathrm{X}+2,312
$$

A Figura 3 apresenta a curva do polinômio da Eq. (9). Observa-se, neste gráfico, uma equação decrescente com domínio entre 1 e 5 e imagem variando entre 0,4 e 1,6.

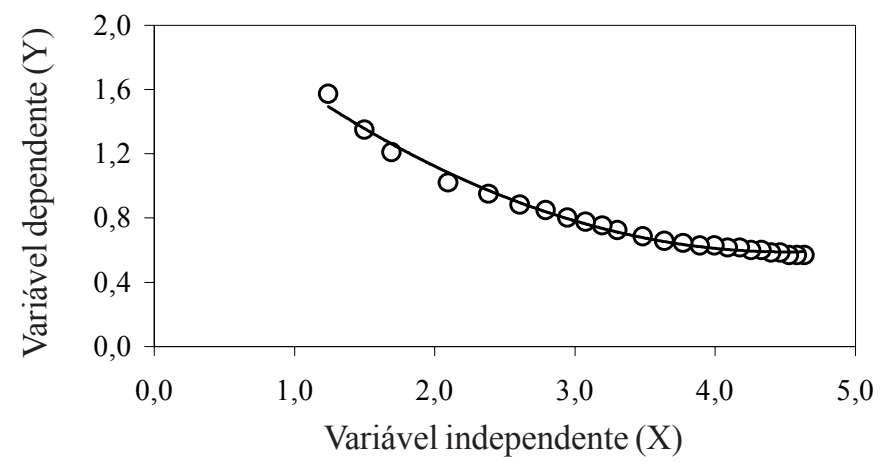

Figura 3. Relação entre as variáveis X e Y

O polinômio do segundo grau apresentado na Eq. (9) fornece os coeficientes de ajuste da Eq. (5). A substituição desses coeficientes na Eq. (5) resulta na Eq. (10) que, por sua vez, representa matematicamente o segundo parâmetro adimensional de forma, do método de Kazemipour.

$\psi_{2}=\exp \left\{\left[0,084\left(1+\ln \mathrm{D} / \mathrm{y}_{\mathrm{D}}\right)^{2}-0,764\left(1+\ln \mathrm{D} / \mathrm{y}_{\mathrm{D}}\right)+2,321\right]-1\right\}$

Aplicando-se propriedades básicas de funções logarítmicas e exponenciais, a Eq. (10) pode ser simplificada, de maneira a se facilitar o cálculo de $\psi_{2}$. A simplificação da Eq. (10) fornece a Eq. (11).

$$
\psi_{2}=1,864 \exp \left[0,084\left(\operatorname{lnD} / \mathrm{y}_{\mathrm{D}}\right)^{2}-0,589\left(\operatorname{lnD} / \mathrm{y}_{\mathrm{D}}\right)\right]
$$

A Figura 4 apresenta os pontos do experimento de Kazemipour utilizados para traçar a curva de $\psi_{2}$, apresentada por Kazemipour \& Apelt (1980) e os valores deste parâmetro obtidos diretamente da Eq. (11).

A Figura 4 revela o forte grau de concordância entre os valores calculados através da Eq. (11) e os dados experimentais utilizados por Kazemipour \& Apelt (1980) para traçar a curva do

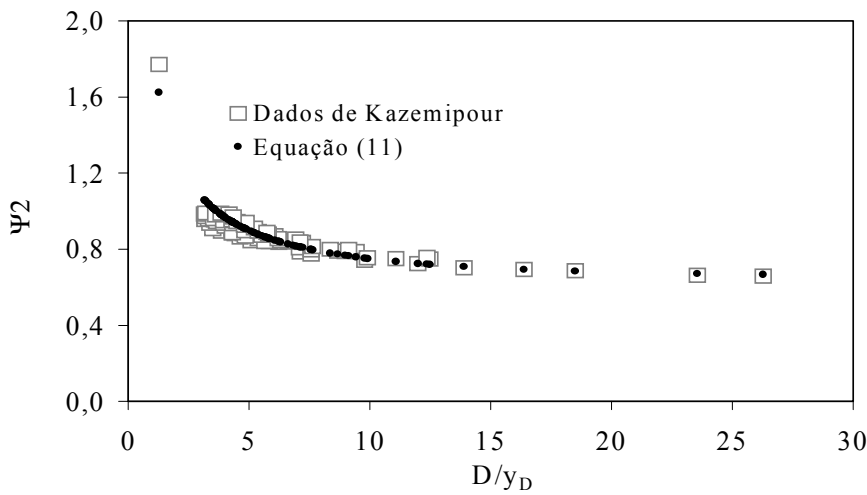

Figura 4. Dados de Kazemipour e Apelt (1980) utilizados para traçar a curva experimental e dados fornecidos pela Eq. (11).

segundo parâmetro de forma, exceto quando os valores de D/ $\mathrm{y}_{\mathrm{D}}$ são maiores que 20; no entanto, valores da relação $\mathrm{D} / \mathrm{y}_{\mathrm{D}}$ superiores a 20 indicam uma altura hidráulica inferior a $5 \%$ do diâmetro interno do canal, valor que, praticamente, não ocorre em casos reais, já que os canais circulares são geralmente dimensionados para trabalharem próximos à altura de eficiência hidráulica máxima ( $50 \%$ do diâmetro).

Desta forma, a boa representação dos dados experimentais através da Eq. (11) indica a possibilidade de utilização desta equação em substituição à curva experimental do segundo parâmetro do método de Kazemipour, no cálculo do escoamento uniforme em canais circulares lisos.

\section{Aplicação do método de Kazemipour adaptado}

Calcular a vazão em um canal circular liso com diâmetro interno de 29,34 cm, altura hidráulica y $=7,34 \mathrm{~cm}$ e declividade do fundo de $0,03409 \mathrm{~m} \mathrm{~m}^{-1}$, que transporta água em escoamento uniforme a uma temperatura de $24,5^{\circ} \mathrm{C}\left(\mathrm{n}=9,00 \times 10^{-3} \mathrm{~cm}^{2} \mathrm{~s}^{-1}\right)$.

Solução:

i) Cálculo da área da seção transversal

$$
\mathrm{A}=132,30 \times 10^{-4} \mathrm{~m}^{2}
$$

ii) Cálculo de $y_{D}$

$$
\mathrm{y}_{\mathrm{D}}=\mathrm{A} / \mathrm{D}=0,0451 \mathrm{~m}
$$

iii) Cálculo de $y_{2}$ (Eq. 11) e obtenção de $\psi$ (Eq. 1 e 2)

$$
\psi=1,238
$$

iv) Através da Eq. (6), calcula-se a parcela $\operatorname{Re}(f)^{1 / 2}$

$$
\operatorname{Re}(\mathrm{f})^{1 / 2}=58280
$$

v) Obtém-se o fator de atrito para tubulações pressurizadas f da Eq. (7)

$$
f=0,0131
$$

vi) Calcula-se o fator de atrito para canais $\mathrm{fc}=\mathrm{f} \psi$

$$
\mathrm{fc}=0,0162
$$


vii) Calcula-se a velocidade média V, Eq. (8)

$$
\mathrm{V}=2,66 \mathrm{~m} \mathrm{~s}^{-1}
$$

viii) Finalmente, calcula-se a vazão $Q$ através do produto da área da seção transversal A pela velocidade média V.

$$
\mathrm{Q}=35,2 \mathrm{~L} \mathrm{~s}^{-1}
$$

Observa-se que o procedimento de solução despendido é um pouco mais laborioso, quando comparado a outras metodologias utilizadas no cálculo do escoamento de canais, a exemplo da equação de Chezy-Manning, mas, atualmente, este problema pode ser contornado com a utilização de rotinas computacionais, elaboradas em microcomputadores ou até em calculadoras programáveis.

\section{CONCLUSÕES}

A partir da análise dos resultados obtidos, chega-se às seguintes conclusões:

1. A Eq. (8), desenvolvida de acordo com o procedimento proposto por Silva \& Figueiredo (1993) para representar o segundo parâmetro adimensional do método de Kazemipour, pode ser utilizada em substituição aos procedimentos gráficos, no cálculo do escoamento turbulento uniforme em canais circulares lisos.

2. O ajuste numérico do método de Kazemipour possibilita a utilização desta metodologia através de uma rotina de computador ou calculadoras programáveis, reduz possíveis erros de leitura, comum nos procedimentos gráficos, e torna capaz a comparação com outras metodologias para cálculo do escoamento uniforme turbulento em canais circulares lisos.

\section{AGRADECIMENTOS}

Ao Professor Dr. Colin J. Apelt, da Universidade de Queensland (Austrália), que gentilmente forneceu dados experimentais imprescindíveis à elaboração deste trabalho, e à Coordenação de Aperfeiçoamento de Pessoal de Nível Superior (CAPES), pela concessão de bolsa de estudo ao primeiro autor.

\section{LITERATURA CITADA}

Goldfarb M.C. Resistência ao escoamento uniforme em canais circulares lisos, Campina Grande: Universidade Federal da Paraíba, 2002. 68p. Dissertação Mestrado

Kazemipour, A.K.; Apelt, C.J. Shape effects on resistance to smooth semi-circular channels. Brisbane: Department of Civil Engineering, University of Queensland, 1980. 26p. Research Report No. CE18

Nalluri, C.; Adepoju, B.A. Shape effects on resistance to flow in smooth channels of circular cross-section. Journal of Hydraulic Research, Delft, Netherlands, v. 23, n.1, p.25-36, 1985.

Oliveira, J.M.S. Resistência ao escoamento uniforme em canais triangulares lisos, João Pessoa: Universidade Federal da Paraíba , 1994. 66p. Dissertação Mestrado

Replogle, J.A; Chow, V.T. Tractive-force distribution in open channels. Journal of the Hydraulics Division, Delft, Netherlands, v.92, n. HY2, p.169-191, 1966.

Silva, T.C. Efeitos de forma na resistência ao escoamento uniforme em canais, São Paulo, SP: Escola Politécnica da Universidade de São Paulo, 1992. 85p. Tese Doutorado

Silva, T.C.; Figueiredo P. H. S. Ajuste polinomial: Três estudos de casos utilizando variáveis transformadas. In: Congresso Ibero Latino-Americano de Métodos Computacionais em Engenharia, 14, 1993, São Paulo, Anais..., São Paulo, 1993. p1212-1219 